\title{
A Home-Made Hartshorne-Serre Correspondence
}

\author{
Enrique ARRONDO
}

\author{
Departamento de Álgebra \\ Facultad de Ciencias Matemáticas \\ Universidad Complutense de Madrid \\ 28040 Madrid - Spain \\ Enrique_Arrondo@mat.ucm.es
}

Received: October 2, 2006

Accepted: March 19, 2007

\begin{abstract}
We provide an elementary proof of the Hartshorne-Serre correspondence for constructing vector bundles from local complete intersection subschemes of codimension two. This will be done, as in the correspondence of hypersurfaces and line bundles, by patching together local determinantal equations in order to produce sections of a vector bundle.
\end{abstract}

Key words: codimension two, Hartshorne-Serre correspondence.

2000 Mathematics Subject Classification: 14M07.

\section{Introduction}

It is well-known that a hypersurface of a smooth algebraic variety can be obtained (in a unique way) as the zero locus of a section of a line bundle. In fact the construction of the line bundle and its section can be done in a very elementary way, by patching local equations and it can be taught in any first course of algebraic geometry.

If instead one considers subvarieties of codimension bigger than one, the situation is very different and only well understood in codimension two. (For some results in codimension three, see $[5,6,15]$.) More precisely, in [8], Hartshorne, inspired by previous works of Serre and Horrocks $([10,13])$, proved that a codimension two subvariety

Supported in part by the funds of the Spanish research project BFM2003-03971. 
of $\mathbb{P}^{n}$ is the zero locus of a rank-two vector bundle over $\mathbb{P}^{n}$ if and only if the subvariety is subcanonical (which can be interpreted as saying that the determinant of its normal bundle extends to a line bundle $L$ on $\mathbb{P}^{n}$ ). This result was independently proved by Barth and Van de Ven ([3]), and generalized by Grauert and Mülich ([7]) to any ambient space (in which case the vanishing of the second order cohomology of $L^{*}$ is needed). Finally, following the original technique of Hartshorne, Vogelaar ([14]) gave the most general result, proving that any local complete intersection subscheme of codimension two of a smooth variety $X$ can be obtained as the dependency locus of $r-1$ sections of a rank $r$ vector bundle over $X$ of determinant $L$ if and only if the determinant of its normal bundle twisted with $L^{*}$ is generated by $r-1$ global sections (provided again the vanishing of the second order cohomology of $L^{*}$ ). In both [7] and [14], the uniqueness of the vector bundle is obtained provided the vanishing of the first order cohomology of $L^{*}$.

However, although this construction (already known as Hartshorne-Serre correspondence) is very well-known and thoroughly used, it is very difficult to provide a good reference of it. Indeed the general result is only in Vogelaar's PhD thesis, which is not published elsewhere, and hence it is usually embarrassing to use as a reference. Even in the subcanonical case, although the technique of [7] works in general, it is written only for the particular case of projective spaces (as it happens for all the other proofs I know of this case).

On the other hand, it is also very annoying that, while the case of codimension one is so easy to explain to even an undergraduate student, the techniques for the case of codimension two are too sophisticated, using in an essential way the spectral sequence of local and global Ext. Only in [7] (which is written in German) there is a more elementary proof of the subcanonical case.

The goal of this paper is hence double. On one hand, we want to provide a reference for the general Hartshorne-Serre correspondence. On the other hand, in order to present some new material, we will give a quite elementary proof of the main result, namely patching together local representations of the sections of the vector bundle we are looking for (hence imitating the standard proof for codimension one). This is in fact the method used in [7], without much details, in the subcanonical case. (I thank the lovely kindness of Sofía Cobo, who translated for me that paper, so that I learned that my first draft [2] contained essentially the proof of [7].) Anyway, the general case still requires some new tricky ideas that we develop in this paper. We also hope that our approach could be extended to other contexts different from algebraic geometry, and maybe give also some idea about how to extend this kind of results to higher codimension.

In a first section, we will recall the main result (Theorem 1.1), and for the sake of completeness we will also recall its standard algebraic proof. This will be the only part in which a good background of algebraic geometry (at the level of [9]) will be required. For the rest of the paper, we hope that it will be readable for a wide range of mathematicians. (It will not be important at all to know what a scheme is!!!) In the 
second section, we will present the minimal background needed to follow the paper.

The proof of Theorem 1.1 will be divided in the next three sections. In section 3 , we will study the main properties that we will require to an open covering of our general ambient variety. In section 4, we will discuss how to construct the $r-1$ sections of the vector bundle we are looking for. We will eventually finish the proof of Theorem 1.1 in section 5 , in which we will see how the cohomological conditions on $L^{*}$ imply the existence and uniqueness of the vector bundle. Finally, we include a last section for some remarks on possible generalizations of the result.

Although I usually do not like to do it, I had to sacrifice the "pedagogical" presentation of the material by the sake of the rigor. In other words, I decided to avoid sentences like "we could have refined our covering so that..." or "changing our definition of... we can assume..." As a result, several definitions and notations that a priori seem artificial can only be understood a posteriori. For example, the strange sign in Lemma 3.1 is explained after Lemma 4.1 (see Remark 4.2), and the apparently complicated way of writing the matrices in section 4 makes sense only in section 5 .

\section{Statement and the standard approach}

Let $X$ be a smooth algebraic variety over an algebraically closed field $k$. Let $Y$ be a codimension two subscheme of $X$. We will denote by $\mathcal{J}$ the ideal sheaf of $Y$ in $X$. If we assume that $Y$ is a local complete intersection, then the conormal sheaf $N^{*}:=\mathcal{J} \otimes \mathcal{O}_{Y}$ is locally free, so that we will regard its dual $N$ as a vector bundle. Assume that $Y$ is the dependency locus of $r-1$ sections $\alpha_{1}, \ldots, \alpha_{r-1}$ of a rank $r$ vector bundle $E$ over $X$ with $\bigwedge^{r} E=L$. This produces an exact sequence

$$
0 \rightarrow(r-1) \mathcal{O}_{X} \stackrel{\left(\alpha_{1}, \ldots, \alpha_{r-1}\right)}{\longrightarrow} E \rightarrow \mathcal{J} \otimes L \rightarrow 0 .
$$

Its restriction to $Y$ produces a long exact sequence

$$
0 \rightarrow \bigwedge^{2} N^{*} \otimes L_{\mid Y} \rightarrow(r-1) \mathcal{O}_{Y} \stackrel{\left(\alpha_{1 \mid Y}, \ldots, \alpha_{r-1 \mid Y}\right)}{\longrightarrow} E_{\mid Y} \rightarrow N^{*} \otimes L_{\mid Y} \rightarrow 0
$$

in which we find out that the kernel of the middle map is $\bigwedge^{2} N^{*} \otimes L_{\mid Y}$ by just looking at the first Chern classes in the sequence. Dualizing the first map in (2) we obtain in particular that the line bundle $\bigwedge^{2} N \otimes L_{\mid Y}^{*}$ is generated by $r-1$ global sections $s_{1}, \ldots, s_{r-1}$ that also satisfy

$$
s_{1} \alpha_{1 \mid Y}+\cdots+s_{r-1} \alpha_{r-1 \mid Y}=0 .
$$

Hartshorne-Serre correspondence consists of reversing this process. More precisely:

Theorem 1.1. Let $X$ be a smooth algebraic variety and let $Y$ be a local complete intersection subscheme of codimension two in $X$. Let $N$ be the normal bundle of $Y$ in $X$ and let $L$ be a line bundle on $X$ such that $H^{2}\left(X, L^{*}\right)=0$. Assume that 
$\bigwedge^{2} N \otimes L_{\mid Y}^{*}$ has $r-1$ generating global sections $s_{1}, \ldots, s_{r-1}$. Then there exists a rank $r$ vector bundle $E$ over $X$ such that:

(i) $\bigwedge^{r} E=L$;

(ii) E has $r-1$ global sections $\alpha_{1}, \ldots, \alpha_{r-1}$ whose dependency locus is $Y$ and such that $s_{1} \alpha_{1 \mid Y}+\cdots+s_{r-1} \alpha_{r-1 \mid Y}=0$.

Moreover, if $H^{1}\left(X, L^{*}\right)=0$, conditions (i) and (ii) determine $E$ up to isomorphism.

The main idea for the standard algebraic proof is to obtain $E$ as an extension like (1), i.e., as a suitable element in $\operatorname{Ext}^{1}\left(\mathcal{J} \otimes L,(r-1) \mathcal{O}_{X}\right)$. For this, one first considers the spectral sequence

$$
\left.E_{2}^{p, q}:=H^{p}\left(\mathcal{E} x t^{q}\left(\mathcal{J} \otimes L,(r-1) \mathcal{O}_{X}\right)\right) \Rightarrow E_{n}:=\operatorname{Ext}^{n}\left(L \otimes \mathcal{J},(r-1) \mathcal{O}_{X}\right)\right)
$$

(see $[1$, Proposition IV(2.4)]). Then the exact sequence

$$
0 \rightarrow E_{2}^{1,0} \rightarrow E^{1} \rightarrow E_{2}^{0,1} \rightarrow E_{2}^{2,0}
$$

(see for instance [12, Theorem 11.43]), becomes, under natural identifications:

$$
\begin{aligned}
0 \rightarrow H^{1}\left(X,(r-1) L^{*}\right) & \left.\rightarrow \operatorname{Ext}^{1}\left(\mathcal{J} \otimes L,(r-1) \mathcal{O}_{X}\right)\right) \stackrel{\varphi}{\longrightarrow} \\
& \stackrel{\varphi}{\longrightarrow} \operatorname{Hom}\left((r-1) \mathcal{O}_{X}, \bigwedge^{2} N \otimes L_{\mid Y}^{*}\right) \stackrel{\psi}{\longrightarrow} H^{2}\left(X,(r-1) L^{*}\right)
\end{aligned}
$$

Under the hypothesis $H^{2}\left(X, L^{*}\right)=0$, the map $\varphi$ is surjective, and hence the element $\eta \in \operatorname{Hom}\left((r-1) \mathcal{O}_{X}, \bigwedge^{2} N \otimes L_{\mid Y}^{*}\right)$ corresponding to the choice of $s_{1}, \ldots, s_{r-1}$ will produce an extension as in (1) (which will be unique if $H^{1}\left(X, L^{*}\right)=0$ ). Hence it is enough to check that $E$ is a locally free sheaf. The proof given in [14] is not clear to us, so that we outline here another (standard) one. We need to show that $\mathcal{E} x t^{i}\left(E, \mathcal{O}_{X}\right)=$ 0 for all $i>0$, and this can be done by applying the functor $\mathcal{H o m}\left(\ldots, \mathcal{O}_{X}\right)$ to the exact sequence (1) we just constructed. The only difficulty is to show the vanishing of $\mathcal{E} x t^{1}\left(E, \mathcal{O}_{X}\right)$, but this follows from the fact that in the exact sequence

$$
\mathcal{H o m}\left((r-1) \mathcal{O}_{X}, \mathcal{O}_{X}\right) \rightarrow \mathcal{E} x t^{1}\left(\mathcal{J} \otimes L, \mathcal{O}_{X}\right) \rightarrow \mathcal{E} x t^{1}\left(E, \mathcal{O}_{X}\right) \rightarrow 0
$$

the first morphism is canonically identified with the surjection $\eta:(r-1) \mathcal{O}_{X} \rightarrow$ $\bigwedge^{2} N \otimes L_{\mid Y}^{*}$ induced by $s_{1}, \ldots, s_{r-1}$.

\section{General background and notations}

We fix $X$ and $Y$ as in Theorem 1.1. If $U$ is an affine subset of $X$, the set $\mathcal{O}_{X}(U)$ of regular functions on $U$ is the coordinate ring of $U$ (considered as an affine subset 
in some affine space). Observe that then the Hilbert Nullstellensatz implies that, for any set of regular functions $f, g \in \mathcal{O}_{X}(U)$, it holds:

$$
\begin{aligned}
\{p \in U \mid f(p)=g(p)=0\} & =\emptyset \\
& \Rightarrow \text { there exist } u, v \in \mathcal{O}_{X}(U) \text { such that } u f+v g=1 .
\end{aligned}
$$

(The same is true for an arbitrary number of functions, but we will not use it.)

The fact that $Y$ is a local complete intersection subscheme of $X$ of codimension two implies (the reader who is not familiar with the theory of schemes can take this as a definition) that any point of $Y$ has an affine open neighborhood $U \subset X$ such that $\mathcal{J}(U)$, the ideal of $Y \cap U$ inside $U$, is generated by two regular functions $f, g \in \mathcal{O}_{X}(U)$ "without common components," i.e., for any regular functions $u, v \in \mathcal{O}_{X}(U)$ it holds:

$$
u f=v g \Rightarrow \text { there exists } w \in \mathcal{O}_{X}(U) \text { such that } u=w g, v=w f .
$$

Instead of regarding vector bundles as locally free sheaves (as we did in the previous section), we will consider their geometric interpretation. Hence, for a vector bundle $E$ of rank $r$ over an algebraic variety $X$ we will take an (affine) open covering $X=$ $\bigcup_{i \in I} U_{i}$ such that $E_{\mid U_{i}} \cong U_{i} \times k^{r}$ (i.e., $E$ trivializes on $U_{i}$ ). For any $i, j \in I$, elements in $E_{\mid U_{i} \cap U_{j}}$ can be regarded as elements in both $U_{j} \times k^{r}$ and $U_{i} \times k^{r}$, and the pass from one to another is given by the multiplication by an $r \times r$ transition matrix $Z_{i j}$ of regular functions on $U_{i} \cap U_{j}$ (when $r=1$, we just speak of the transition functions of the line bundle). Hence a vector bundle can be characterized by a collection of matrices $\left\{Z_{i j}\right\}_{i, j \in I}$ subject to the compatibility condition $Z_{i k}=Z_{i j} Z_{j k}$ (and $Z_{i i}=I_{r}$, the identity matrix).

If $U$ is an affine set of $X$, then $Y \cap U$ is also affine, and hence $\mathcal{O}_{Y}(Y \cap U)=$ $\mathcal{O}_{X}(U) /(f, g)$. We will always use a bar to indicate the classes of elements (vector bundles, functions, matrices...) modulo $Y$ (or any $Y \cap U$ ).

\section{Affine coverings of $X$}

We start taking a covering of $Y$ by affine sets $Y \cap U_{i}$ (with $i$ varying in a set $I$ ) such that:

(i) $U_{i}$ is an affine set of $X$.

(ii) The vector bundle $L$ trivializes on $U_{i}$ and has transition functions $h_{i j}$.

(iii) $\mathcal{J}\left(U_{i}\right)$ is generated by the vanishing of two regular functions $f_{i}, g_{i}$ on $U_{i}$.

In the intersection of two of those open sets, $U_{i}, U_{j}$ we have now two different sets of generators for the ideal $\mathcal{J}\left(U_{i} \cap U_{j}\right)$, and hence it is possible to find a matrix $A_{i j}$ (not necessarily unique) satisfying:

$$
\left(\begin{array}{l}
f_{i} \\
g_{i}
\end{array}\right)=A_{i j}\left(\begin{array}{l}
f_{j} \\
g_{j}
\end{array}\right)=\left(\begin{array}{ll}
a_{i j} & b_{i j} \\
c_{i j} & d_{i j}
\end{array}\right)\left(\begin{array}{l}
f_{j} \\
g_{j}
\end{array}\right)
$$


where $a_{i j}, b_{i j}, c_{i j}, d_{i j}$ are regular functions on $U_{i} \cap U_{j}$ and $\operatorname{det} A_{i j}$ does not have zeros on $U_{i} \cap U_{j}$. Observe that it could happen that $Y \cap U_{i} \cap U_{j}=\emptyset$. In this case, by (4), we can find $u_{i}, v_{i}, u_{j}, v_{j}$ such that $u_{i} f_{i}+v_{i} g_{i}=1=u_{j} f_{j}+v_{j} g_{j}$, and thus we can take $A_{i j}=\left(\begin{array}{cc}f_{i} & -v_{i} \\ g_{i} & u_{i}\end{array}\right)\left(\begin{array}{cc}u_{j} & v_{j} \\ -g_{j} & f_{j}\end{array}\right)$.

Observe also that the vector bundle $N$ trivializes on $Y \cap U_{i}$ and has as transition matrices the restriction $\bar{A}_{i j}$ of $A_{i j}$ to $Y \cap U_{i} \cap U_{j}$.

Let $s_{1}, \ldots, s_{r-1}$ be the global sections generating $\bigwedge^{2} N \otimes L^{*}$. For $t=1, \ldots, r-1$, the section $s_{t}$ can be represented locally at each $Y \cap U_{i}$ by a regular function $\bar{s}_{i t}$ such that there are relations

$$
\bar{s}_{i t}=\frac{\operatorname{det} \bar{A}_{i j}}{\bar{h}_{i j}} \bar{s}_{j t}
$$

Since $\bar{s}_{i 1}, \ldots, \bar{s}_{i, r-1}$ do not vanish simultaneously on $Y \cap U_{i}$, we can refine the covering and assume that there is $t_{i} \in\{1, \ldots, r-1\}$ such that $\bar{s}_{i t_{i}}$ does not have zeros in $Y \cap U_{i}$. Replacing $U_{i}$ with its intersection with $\left\{s_{i t_{i}} \neq 0\right\}$, we can assume that $s_{i t_{i}}$ does not have zeros in $U_{i}$, i.e., it is a unit in $\mathcal{O}_{X}\left(U_{i}\right)$.

Lemma 3.1. With the above notations, it is possible to choose regular functions $f_{i}$, $g_{i}$ such that $s_{i t_{i}}=(-1)^{t_{i}}$. In particular, $\operatorname{det} \bar{A}_{i j}=(-1)^{t_{i}} \frac{\bar{h}_{i j}}{\bar{s}_{j t_{i}}}$.

Proof. We choose as a new set of generators of each $\mathcal{J}\left(U_{i}\right)$ the functions $f_{i}^{\prime}=\frac{f_{i}}{s_{i t_{i}}}$ and $g_{i}^{\prime}=(-1)^{t_{i}} g_{i}$. We obtain a new relation like (6) with a new matrix $A_{i j}^{\prime}$ :

$$
\left(\begin{array}{l}
f_{i}^{\prime} \\
g_{i}^{\prime}
\end{array}\right)=A_{i j}^{\prime}\left(\begin{array}{l}
f_{j}^{\prime} \\
g_{j}^{\prime}
\end{array}\right)=\left(\begin{array}{cc}
\frac{s_{j t_{j}}}{s_{i t_{i}}} a_{i j} & \frac{(-1)^{-t_{j}}}{s_{i t_{i}}} b_{i j} \\
(-1)^{t_{i}} c_{i j} s_{j t_{j}} & (-1)^{t_{i}-t_{j}} d_{i j}
\end{array}\right)\left(\begin{array}{l}
f_{j}^{\prime} \\
g_{j}^{\prime}
\end{array}\right)
$$

from which we get, by (7),

$$
(-1)^{t_{i}} \frac{\bar{s}_{i t}}{\bar{s}_{i t_{i}}}=(-1)^{t_{j}} \frac{\operatorname{det} \bar{A}_{i j}^{\prime}}{\bar{h}_{i j}} \frac{\bar{s}_{j t}}{\bar{s}_{j t_{j}}} .
$$

This shows that, with this new choice of $f_{i}^{\prime}, g_{i}^{\prime}$, the sections $s_{1}, \ldots, s_{r-1}$ can be represented in $Y \cap U_{i}$ by the classes of $(-1)^{t_{i}} \frac{s_{i 1}}{s_{i t_{i}}}, \ldots,(-1)^{t_{i}} \frac{s_{i, r-1}}{s_{i t_{i}}}$. This implies that we can assume $s_{i t_{i}}=(-1)^{t_{i}}$. With this choice, the last statement is just (7) applied to $t=t_{i}$.

We extend now the affine covering to a covering of the whole $X$. For this, we have to cover $X \backslash Y$ by new affine open sets $U_{i}$. For such a new open set we take $f_{i}=1, g_{i}=0$. Observe that, even if $Y \cap U_{i}=\emptyset$, property (5) still holds in a trivial way.

We also have matrices $A_{i j}$ as in (6) for any choice of open sets $U_{i}, U_{j}$. Specifically:

- If $Y \cap U_{i} \neq \emptyset \neq Y \cap U_{j}$, we do as in (6). 
- If $Y \cap U_{i}=\emptyset=Y \cap U_{j}$, we take $A_{i j}$ to be the identity matrix.

- If $Y \cap U_{i} \neq \emptyset=Y \cap U_{j}$, we take $A_{i j}=\left(\begin{array}{cc}u_{j} & v_{j} \\ -g_{j} & f_{j}\end{array}\right)$, with $u_{j}, v_{j}$ such that $u_{j} f_{j}+$ $v_{j} f_{j}=1$.

- If $Y \cap U_{i}=\emptyset \neq Y \cap U_{j}$, we take $A_{i j}=\left(\begin{array}{cc}f_{i} & -v_{i} \\ g_{i} & u_{i}\end{array}\right)$, with $u_{i}, v_{i}$ such that $u_{i} f_{i}+$ $v_{i} f_{i}=1$.

Lemma 3.2. With the above choices and notations, it is possible to choose the matrices $A_{i j}$ such that $\operatorname{det} A_{i j}=(-1)^{t_{i}} \frac{h_{i j}}{s_{j t_{i}}}$.

Proof. By Lemma 3.1, on each $U_{i} \cap U_{j}$ the regular functions $\operatorname{det} A_{i j}$ and $(-1)^{t_{i}} \frac{h_{i j}}{s_{j t_{i}}}$ coincide modulo the ideal $\left(f_{i}, g_{i}\right)$ (this is trivial if $\left.Y \cap U_{i}=\emptyset\right)$. We can thus write

$(-1)^{t_{i}} \frac{h_{i j}}{s_{j t_{i}}}=\operatorname{det} A_{i j}+\varphi_{i j} f_{i}+\psi_{i j} g_{i}=\operatorname{det} A_{i j}+\left(\varphi_{i j} a_{i j}+\psi_{i j} c_{i j}\right) f_{j}+\left(\varphi_{i j} b_{i j}+\psi_{i j} d_{i j}\right) g_{j}$

for some regular functions $\varphi_{i j}, \psi_{i j}$ on $U_{i} \cap U_{j}$. Therefore we can replace (6) with

$$
\left(\begin{array}{c}
f_{i} \\
g_{i}
\end{array}\right)=\left(\begin{array}{ll}
a_{i j}+\psi_{i j} g_{j} & b_{i j}-\psi_{i j} f_{j} \\
c_{i j}-\varphi_{i j} g_{j} & d_{i j}+\varphi_{i j} f_{j}
\end{array}\right)\left(\begin{array}{c}
f_{j} \\
g_{j}
\end{array}\right)
$$

and the new transition matrix $A_{i j}^{\prime}=\left(\begin{array}{ll}a_{i j}+\psi_{i j} g_{j} & b_{i j}-\psi_{i j} f_{j} \\ c_{i j}-\varphi_{i j} g_{j} & d_{i j}+\varphi_{i j} f_{j}\end{array}\right)$ satisfies the wanted property $\operatorname{det} A_{i j}^{\prime}=(-1)^{t_{i}} \frac{h_{i j}}{s_{j t_{i}}}$.

\section{Constructing the sections}

We start by fixing a notation that we will use in the rest of the paper.

Notation. Given the identity matrix (whose order will be clear any time from the context), we will denote by $\Delta_{t}$ the submatrix obtained by removing its $t$-th row. Hence, for any matrix $M$, the matrix $\Delta_{t} M$ will be the submatrix of $M$ obtained by removing its $t$-th row. Similarly, if $\Delta_{t}^{\prime}$ is the transpose of $\Delta_{t}$, then $M \Delta_{t}^{\prime}$ will be the submatrix of $M$ obtained by removing its $t$-th column.

Before constructing the vector bundle $E$ and its $r-1$ sections $\alpha_{1}, \ldots, \alpha_{r-1}$, let us assume that they exist and see the form they can take. Assume, without loss of generality, that $E$ trivializes on each $U_{i}$. Since $s_{1} \alpha_{1 \mid Y}+\cdots+s_{r-1} \alpha_{r-1 \mid Y}=0$ and $s_{t_{i}}$ is represented by $(-1)^{t_{i}}$ on $Y \cap U_{i}$, this means that, on the points of $Y, \alpha_{t_{i}}$ depends on $\alpha_{1}, \ldots, \hat{\alpha}_{t_{i}}, \ldots, \alpha_{r-1}$. (We use the standard notation of a hat to indicate that a term is removed.) Since the rank of $\alpha_{1}, \ldots, \alpha_{r-1}$ is $r-2$ on $Y$ and $r-1$ outside $Y$, it follows that $\alpha_{1}, \ldots, \hat{\alpha}_{t_{i}}, \ldots, \alpha_{r-1}$ are linearly independent on $U_{i}$. Extending them 
to a basis of $E_{\mid U_{i}}$, it is then possible to represent $\alpha_{1}, \ldots, \alpha_{r-1}$ on $U_{i}$, in terms of this basis, as the columns of an $r \times(r-2)$ matrix $M_{i}=\Delta_{t_{i}} T_{i}$, where

$$
T_{i}=\left(\begin{array}{cccccc}
1 & 0 & \ldots & \alpha_{i 1} & \ldots & 0 \\
0 & 1 & \ldots & \alpha_{i 2} & \ldots & 0 \\
\vdots & & \ddots & \vdots & & \vdots \\
0 & 0 & \ldots & \alpha_{i t_{i}} & \ldots & 0 \\
\vdots & & & \vdots & \ddots & \vdots \\
0 & 0 & \ldots & \alpha_{i, r-1} & \ldots & 1 \\
0 & 0 & \ldots & \alpha_{i r} & \ldots & 0 \\
0 & 0 & \ldots & \alpha_{i, r+1} & \ldots & 0
\end{array}\right)
$$

Since $Y \cap U_{i}$ must the determinantal variety defined by the maximal minors of $M_{i}$, it follows that $\alpha_{i r}, \alpha_{i, r+1}$ generate $\mathcal{J}\left(U_{i}\right)$. Hence, changing the last two rows of $T_{i}$ by a suitable linear combination of them, we can assume $\alpha_{i r}=f_{i}, \alpha_{i, r+1}=g_{i}$.

On the other hand, the equation $s_{1} \alpha_{1 \mid Y}+\cdots+s_{r-1} \alpha_{r-1 \mid Y}=0$ implies that the entries of

$$
M_{i}\left(\begin{array}{c}
s_{i 1} \\
\vdots \\
s_{i, r-1}
\end{array}\right)
$$

are a linear combination of $f_{i}, g_{i}$. Hence, after adding to each of the first $r-2$ rows of $M_{i}$ a linear combination of the last two, we can take

$$
T_{i}=\left(\begin{array}{c}
T_{i}^{\prime} \\
T_{i}^{\prime \prime}
\end{array}\right)
$$

with

$$
T_{i}^{\prime}=\left(\begin{array}{cccccc}
1 & 0 & \ldots & -(-1)^{t_{i}} s_{i 1} & \ldots & 0 \\
0 & 1 & \ldots & -(-1)^{t_{i}} s_{i 2} & \ldots & 0 \\
\vdots & & \ddots & \vdots & & \vdots \\
0 & 0 & \ldots & 1 & \ldots & 0 \\
\vdots & & & \vdots & \ddots & \vdots \\
0 & 0 & \ldots & -(-1)^{t_{i}} s_{i, r-1} & \ldots & 1
\end{array}\right)
$$

and

$$
T_{i}^{\prime \prime}=\left(\begin{array}{cccccc}
0 & 0 & \ldots & f_{i} & \ldots & 0 \\
0 & 0 & \ldots & g_{i} & \ldots & 0
\end{array}\right)
$$

We will thus define

$$
M_{i}=\left(\begin{array}{c}
\Delta_{t_{i}} T_{i}^{\prime} \\
T_{i}^{\prime \prime}
\end{array}\right)
$$


with $T_{i}^{\prime}$ and $T_{i}^{\prime \prime}$ as in (8) and (9). We have the following easy equalities, which we will use frequently:

$$
\begin{gathered}
\Delta_{t_{i}} T_{i}^{\prime} \Delta_{t_{i}}^{\prime}=I_{r-2}, \\
T_{i}^{\prime \prime} \Delta_{t_{i}}^{\prime}=\left(\begin{array}{ccc}
0 & \ldots & 0 \\
0 & \ldots & 0
\end{array}\right), \\
\Delta_{t_{i}} T_{i}^{\prime}\left(\begin{array}{c}
s_{i 1} \\
\vdots \\
s_{i, r-1}
\end{array}\right)=\left(\begin{array}{c}
0 \\
\vdots \\
0
\end{array}\right), \\
T_{i}^{\prime \prime}\left(\begin{array}{c}
s_{i 1} \\
\vdots \\
s_{i, r-1}
\end{array}\right)=(-1)^{t_{i}}\left(\begin{array}{c}
f_{i} \\
g_{i}
\end{array}\right) .
\end{gathered}
$$

Since we want the columns of $M_{i}$ to represent the sections $\alpha_{1}, \ldots, \alpha_{r-1}$ of a vector bundle $E$, we need to find the transition matrices relating $M_{i}$ to $M_{j}$. The next result provides a first condition to find them.

Lemma 4.1. For a covering and choices as in Lemma 3.2, if for each $i \in I$ we take $M_{i}$ as in (10), then an $r \times r$ matrix $Z_{i j}=\left(\begin{array}{cc}P_{i j} & Q_{i j} \\ R_{i j} & S_{i j}\end{array}\right)$ satisfies the equality $M_{i}=Z_{i j} M_{j}$ if and only if the following equalities hold:

(i) $P_{i j}=\Delta_{t_{i}} T_{i}^{\prime} \Delta_{t_{j}}^{\prime}$

(ii) $R_{i j}=T_{i}^{\prime \prime} \Delta_{t_{j}}^{\prime}$

(iii) $Q_{i j}\left(\begin{array}{c}f_{j} \\ g_{j}\end{array}\right)=(-1)^{t_{j}} \Delta_{t_{i}} T_{i}^{\prime}\left(\begin{array}{c}s_{j 1} \\ \vdots \\ s_{j, r-1}\end{array}\right)$

(iv) $S_{i j}\left(\begin{array}{c}f_{j} \\ g_{j}\end{array}\right)=(-1)^{t_{j}} s_{j t_{i}}\left(\begin{array}{c}f_{i} \\ g_{i}\end{array}\right)$, i.e., $S_{i j}=(-1)^{t_{j}} s_{j t_{i}} A_{i j}$, with $A_{i j}$ as in (6).

Moreover, such a matrix always exists and, when taking $A_{i j}$ as in Lemma 3.2, it follows $\operatorname{det} S_{i j}=(-1)^{t_{i}} s_{j t_{i}} h_{i j}$ and $\operatorname{det} Z_{i j}=h_{i j}$.

Proof. We have to find the solutions of

$$
\begin{cases}\Delta_{t_{i}} T_{i}^{\prime}= & P_{i j} \Delta_{t_{j}} T_{j}^{\prime}+Q_{i j} T_{j}^{\prime \prime} \\ T_{i}^{\prime \prime}= & R_{i j} \Delta_{t_{j}} T_{j}^{\prime}+S_{i j} T_{j}^{\prime \prime}\end{cases}
$$

Multiplying by $\Delta_{t_{j}}^{\prime}$ to the right the two equations in (15) (i.e., removing the $t_{j}$-th columns of all the terms), we get from (11) and (12) the equalities (i) and (ii). It remains to characterize when (15) holds for the $t_{j}$-th column of each term. To see 
this, since $s_{j t_{j}}=(-1)^{t_{j}}$, it is equivalent to consider the product of the two equalities of (15) with

$$
\left(\begin{array}{c}
s_{j 1} \\
\vdots \\
s_{j, r-1}
\end{array}\right)
$$

which together with (13) and (14) yield exactly the equalities (iii) and (iv).

The entries of

$$
\Delta_{t_{i}} T_{i}^{\prime}\left(\begin{array}{c}
s_{j 1} \\
\vdots \\
s_{j, r-1}
\end{array}\right)
$$

are $s_{j t}-(-1)^{t_{i}} s_{i t} s_{j t_{i}}$, with $t=1, \ldots, \hat{t}_{i}, \ldots, r-1$. Recalling from Lemma 3.2 that $\operatorname{det} A_{i j}=(-1)^{t_{i}} \frac{h_{i j}}{s_{j t_{i}}}$, equality (7) reads $\bar{s}_{j t}-(-1)^{t_{i}} \bar{s}_{i t} \bar{s}_{j t_{i}}=\overline{0}$. Hence the entries of (16) are in the ideal $\left(f_{j}, g_{j}\right)$ defining $Y \cap U_{i} \cap U_{j}$, and the same holds clearly for the entries of $s_{j t_{i}}\left(\begin{array}{c}f_{i} \\ g_{i}\end{array}\right)$. Therefore, equalities (iii) and (iv) have solutions $Q_{i j}, S_{i j}$, and thus there exists some $Z_{i j}$ such that $M_{i}=Z_{i j} M_{j}$.

For the last equality in the statement, we deduce from the equations (15) multiplied to the right by $\Delta_{t_{i}}^{\prime}$, and using (11) and (12), the equality

$$
\left(\begin{array}{cc}
P_{i j} & Q_{i j} \\
R_{i j} & S_{i j}
\end{array}\right)\left(\begin{array}{cc}
\Delta_{t_{j}} T_{j}^{\prime} \Delta_{t_{i}}^{\prime} & 0 \\
T_{j}^{\prime \prime} \Delta_{t_{i}}^{\prime} & I_{2}
\end{array}\right)=\left(\begin{array}{cc}
I_{r-2} & Q_{i j} \\
0 & S_{i j}
\end{array}\right) .
$$

Hence, observing that $\operatorname{det}\left(\Delta_{t_{j}} T_{j}^{\prime} \Delta_{t_{i}}^{\prime}\right)=(-1)^{t_{i}} s_{j t_{i}}$, we obtain $(-1)^{t_{i}} s_{j t_{i}} \operatorname{det} Z_{i j}=$ $\operatorname{det} S_{i j}$. Since $S_{i j}=(-1)^{t_{i}} s_{j t_{i}} A_{i j}$ and $\operatorname{det} A_{i j}=(-1)^{t_{j}} \frac{h_{i j}}{s_{j t_{i}}}$ after Lemma 3.2, we thus have $\operatorname{det} S_{i j}=(-1)^{t_{i}} s_{j t_{i}} h_{i j}$ and therefore $\operatorname{det} Z_{i j}=h_{i j}$.

Remark 4.2. It is only now that one can understand the reason of introducing the sign $(-1)^{t_{i}}$ in Lemma 3.1. Observe first that it was not a misprint to write $\operatorname{det}\left(\Delta_{t_{j}} T_{j}^{\prime} \Delta_{t_{i}}^{\prime}\right)=$ $(-1)^{t_{i}} s_{j t_{i}}$ at the end of the proof of Lemma 4.1, in the sense that it is indeed $(-1)^{t_{i}}$ instead of $(-1)^{t_{j}}$ (which is the sign appearing in the entries of the matrix $T_{j}^{\prime}$ ). If we had not included that sign in Lemma 3.1, we would have obtained now $\operatorname{det} Z_{i j}=$ $(-1)^{t_{i}+t_{j}} h_{i j}$ in Lemma 4.1. This would not have been a disaster, since the functions $(-1)^{t_{i}+t_{j}} h_{i j}$ are also transition functions of $L$. Anyway, we thought it was more elegant and clearer not to work simultaneously with two different sets of transition functions of the same line bundle.

Lemma 4.3. For a matrix $Z_{i j}$ as in Lemma 4.1, the following equalities hold:

(i) $\left(g_{i},-f_{i}\right) S_{i j}=(-1)^{t_{i}+t_{j}} h_{i j}\left(g_{j},-f_{j}\right)$

(ii) $R_{i j}=\left(\begin{array}{c}f_{i} \\ g_{i}\end{array}\right)\left(\delta_{i j 1} \ldots \hat{\delta}_{i j t_{j}} \ldots \delta_{i j, r-1}\right)$, with $\delta_{i j t}=0$, for all $t \neq t_{i}$ and $\delta_{i j t_{i}}=1$; in particular, $\left(g_{i},-f_{i}\right) R_{i j}=(0 \ldots 0)$. 
(iii) $\left(0 \ldots 0 g_{i},-f_{i}\right) Z_{i j}=(-1)^{t_{i}+t_{j}} h_{i j}\left(0 \ldots 0 g_{j},-f_{j}\right)$.

(iv) $\left(0 \ldots, 0 g_{i},-f_{i}\right)\left(Z_{i j} Z_{j k}-Z_{i k}\right)=(0 \ldots 0)$.

Proof. The equality $S_{i j}\left(\begin{array}{c}f_{j} \\ g_{j}\end{array}\right)=(-1)^{t_{j}} s_{j t_{i}}\left(\begin{array}{c}f_{i} \\ g_{i}\end{array}\right)$ of Lemma 4.1 is equivalent, multiplying to the left by $\operatorname{Adj} S_{i j}$ and using $\operatorname{det} S_{i j}=(-1)^{t_{i}} s_{j t_{i}} h_{i j}$, to $\left(\begin{array}{c}f_{j} \\ g_{j}\end{array}\right)=\frac{(-1)^{t_{i}+t_{j}}}{h_{i j}} \operatorname{Adj} S_{i j}\left(\begin{array}{c}f_{i} \\ g_{i}\end{array}\right)$, which is in turn equivalent to (i). Part (ii) is obvious, since $R_{i j}=T_{i}^{\prime \prime} \Delta_{t_{j}}^{\prime}$. Part (iii) follows from (i) and (ii). Finally, part (iv) is a consequence of (iii), having in mind, by Lemma 4.1 , that the $h_{i j}$ are the transition functions of the line bundle $L$ and therefore $h_{i j} h_{j k}=h_{i k}$.

Corollary 4.4. If the matrices $\left\{Z_{i j}\right\}_{i, j, \in I}$ are chosen as in Lemma 4.1, then for any $i, j, k \in I$ there exist regular functions $\beta_{i j k 1}, \ldots, \beta_{i j k, r-1}$ on $U_{i} \cap U_{j} \cap U_{k}$ such that $Z_{i k}-Z_{i j} Z_{j k}=\left(\begin{array}{ll}0 & B_{i j k}\end{array}\right)$, with

$$
B_{i j k}=\left(\begin{array}{c}
Q_{i k}-P_{i j} Q_{j k}-Q_{i j} S_{j k} \\
S_{i k}-R_{i j} Q_{j k}-S_{i j} S_{j k}
\end{array}\right)=\left(\begin{array}{c}
\beta_{i j k 1} \\
\vdots \\
\hat{\beta}_{i j k t_{i}} \\
\vdots \\
\beta_{i j k, r-1} \\
\beta_{i j k t_{i}} f_{i} \\
\beta_{i j k t_{i}} g_{i}
\end{array}\right)\left(g_{k},-f_{k}\right)
$$

Proof. Write $Z_{i k}-Z_{i j} Z_{j k}=\left(B_{i j k}^{\prime} B_{i j k}^{\prime \prime}\right)$. The equality $\left(Z_{i k}-Z_{i j} Z_{j k}\right) M_{k}=0$ is equivalent to $B_{i j k}^{\prime} \Delta_{t_{k}} T_{k}^{\prime}+B_{i j k}^{\prime \prime} T_{k}^{\prime \prime}=0$, so it follows, multiplying this equality to the right by $\Delta_{t_{k}}^{\prime}$ and applying (11) and (12), that $B_{i j k}^{\prime}=0$. Hence $B_{i j k}^{\prime \prime} T_{k}^{\prime \prime}=0$, i.e., by the definition (9) of $T_{k}^{\prime \prime}, B_{i j k}^{\prime \prime}\left(\begin{array}{c}f_{k} \\ f g_{k}\end{array}\right)=0$. It follows from (5) that there exist regular functions $\beta_{i j k 1}, \ldots, \hat{\beta}_{i j k t_{i}}, \ldots, \beta_{i j k, r+1}$ such that

$$
B_{i j k}=\left(\begin{array}{c}
\beta_{i j k 1} \\
\vdots \\
\hat{\beta}_{i j k t_{i}} \\
\vdots \\
\beta_{i j k, r+1}
\end{array}\right)\left(g_{k},-f_{k}\right) .
$$

On the other hand, applying now Lemma 4.3 (iv), we get $\left(g_{i},-f_{i}\right)\left(\begin{array}{c}\beta_{i j k r} \\ \beta_{i j k, r+1}\end{array}\right)=0$, from which the lemma follows by applying (5) again.

Remark 4.5. $Z_{i j}$ to be the transition matrices of a vector bundle $E$, we need to find a good choice of $Q_{i j}, S_{i j}$ such that $\beta_{i j k 1}, \ldots, \beta_{i j k, r-1}$ are all zero. Observe that another 
choice of $Q_{i j}^{\prime}$ and $S_{i j}^{\prime}$ satisfies conditions (iii) and (iv) of Lemma 4.1 if and only if we have respectively

$$
\left(Q_{i j}^{\prime}-Q_{i j}\right)\left(\begin{array}{c}
f_{j} \\
g_{j}
\end{array}\right)=\left(\begin{array}{c}
0 \\
\vdots \\
0
\end{array}\right)
$$

and

$$
\left(S_{i j}^{\prime}-S_{i j}\right)\left(\begin{array}{l}
f_{j} \\
g_{j}
\end{array}\right)=\left(\begin{array}{l}
0 \\
0
\end{array}\right) \text {. }
$$

Moreover, using Lemma 4.3 (i), we would also have $\left(g_{i},-f_{i}\right)\left(S_{i j}^{\prime}-S_{i j}\right)=\left(\begin{array}{l}0 \\ 0\end{array}\right)$. Hence the same reasoning as in the proof of Corollary 4.4 shows that the above conditions are equivalent to the existence of regular functions $x_{i j 1}, \ldots, x_{i j, r-1}$ such that

$$
Q_{i j}^{\prime}=Q_{i j}+\left(\begin{array}{c}
x_{i j 1} \\
\vdots \\
\hat{x}_{i j t_{i}} \\
\vdots \\
x_{i j, r-1}
\end{array}\right)\left(g_{j},-f_{j}\right)
$$

and $S_{i j}^{\prime}=S_{i j}+x_{i j t_{i}}\left(\begin{array}{c}f_{i} \\ g_{i}\end{array}\right)\left(g_{j},-f_{j}\right)$. The goal of the next section will be to see that there is essentially one way of choosing the functions $x_{i j 1}, \ldots, x_{i j, r-1}$ on each $U_{i} \cap U_{j}$. We will then see how the a priori strange choice of subindices makes perfectly sense.

\section{Constructing the vector bundle}

We finally find under which conditions the matrices $Z_{i j}$ are transition matrices of a vector bundle. We start with a technical lemma that will be very useful in the sequel:

Lemma 5.1. With the definitions of the previous section, for any vector

$$
u=\left(\begin{array}{c}
u_{1} \\
\vdots \\
u_{r-1}
\end{array}\right),
$$

we have

$$
T_{j}^{\prime-1} u=\Delta_{t_{j}}^{\prime}\left(\begin{array}{c}
u_{1} \\
\vdots \\
\hat{u}_{t_{j}} \\
\vdots \\
u_{r-1}
\end{array}\right)+(-1)^{t_{j}} u_{t_{j}}\left(\begin{array}{c}
s_{j 1} \\
\vdots \\
s_{j, r-1}
\end{array}\right) .
$$

Hence, if we define $u^{\prime}=T_{i}^{\prime} T_{j}^{\prime-1} u$, then: 
(i) $\Delta_{t_{i}} u^{\prime}=P_{i j}\left(\begin{array}{c}u_{1} \\ \vdots \\ \hat{u}_{t_{j}} \\ \vdots \\ u_{r-1}\end{array}\right)+(-1)^{t_{j}} u_{t_{j}} \Delta_{t_{i}} T_{i}^{\prime}\left(\begin{array}{c}s_{j 1} \\ \vdots \\ s_{j, r-1}\end{array}\right)$.

(ii) The $t_{i}$-th row of $u^{\prime}$ is

$$
\left(\delta_{i j 1} \ldots \hat{\delta}_{i j t_{j}} \ldots \delta_{i j, r-1}\right)\left(\begin{array}{c}
u_{1} \\
\vdots \\
\hat{u}_{t_{j}} \\
\vdots \\
u_{r-1}
\end{array}\right)+(-1)^{t_{j}} s_{j t_{i}} u_{t_{j}} .
$$

Proof. For the first equality, observe first that we can write

$$
u=\Delta_{t_{j}}^{\prime}\left(\begin{array}{c}
u_{1} \\
\vdots \\
\hat{u}_{t_{j}} \\
\vdots \\
u_{r-1}
\end{array}\right)+u_{t_{j}}\left(\begin{array}{c}
0 \\
\vdots \\
1 \\
\vdots \\
0
\end{array}\right)
$$

(The first summand is nothing but $u$ with the $t_{j}$-th row replaced with 0 .) Then the wanted equality follows because

$$
T_{j}^{\prime-1}=\left(\begin{array}{cccccc}
1 & 0 & \ldots & (-1)^{t_{j}} s_{j 1} & \ldots & 0 \\
0 & 1 & \ldots & (-1)^{t_{j}} s_{j 2} & \ldots & 0 \\
\vdots & & \ddots & \vdots & & \vdots \\
0 & 0 & \ldots & 1 & \ldots & 0 \\
\vdots & & & \vdots & \ddots & \vdots \\
0 & 0 & \ldots & (-1)^{t_{j}} s_{j, r-1} & \ldots & 1
\end{array}\right)
$$

and then $T_{j}^{\prime-1} \Delta_{t_{j}}^{\prime}=\Delta_{t_{j}}^{\prime}$ (observe also that $(-1)^{t_{j}} s_{j t_{j}}=1$ ) .

Now (i) and (ii) are easy consequences of the first equality: for (i) it is enough to recall from Lemma 4.1 that $P_{i j}=\Delta_{t_{i}} T_{i}^{\prime} \Delta_{t_{j}}^{\prime}$, while for (ii) it suffices to observe that the $t_{i}$-th row of $\Delta_{t_{j}}^{\prime}$ is $\left(\delta_{i j 1} \ldots \hat{\delta}_{i j t_{j}} \ldots \delta_{i j, r-1}\right)$. 
Proposition 5.2. For a choice of matrices $Z_{i j}$ as in Lemma 4.1, let $Z_{i j}^{\prime}=\left(\begin{array}{cc}P_{i j} & Q_{i j}^{\prime} \\ R_{i j} & S_{i j}^{\prime}\end{array}\right)$ with

$$
Q_{i j}^{\prime}=Q_{i j}+\left(\begin{array}{c}
x_{i j 1} \\
\vdots \\
\hat{x}_{i j t_{i}} \\
\vdots \\
x_{i j, r-1}
\end{array}\right)\left(g_{j},-f_{j}\right)
$$

and $S_{i j}^{\prime}=S_{i j}+x_{i j t_{i}}\left(\begin{array}{c}f_{i} \\ g_{i}\end{array}\right)\left(g_{j},-f_{j}\right)$. Then $Z_{i k}^{\prime}-Z_{i j}^{\prime} Z_{j k}^{\prime}=0$ if and only if

$$
\begin{aligned}
(-1)^{t_{k}}{T_{i}^{\prime}}^{-1}\left(\begin{array}{c}
\beta_{i j k 1} \\
\vdots \\
\beta_{i j k, r-1}
\end{array}\right) & =(-1)^{t_{k}}{T_{j}^{\prime}}^{-1}\left(\begin{array}{c}
x_{j k 1} \\
\vdots \\
x_{j k, r-1}
\end{array}\right)- \\
& -(-1)^{t_{k}}{T_{i}^{\prime}}^{-1}\left(\begin{array}{c}
x_{i k 1} \\
\vdots \\
x_{i k, r-1}
\end{array}\right)+(-1)^{t_{j}} h_{j k} T_{i}^{\prime-1}\left(\begin{array}{c}
x_{i j 1} \\
\vdots \\
x_{i j, r-1}
\end{array}\right) .
\end{aligned}
$$

Proof. Multiplying to the left by $(-1)^{t_{k}} T_{i}^{\prime}$, equation (17) in the statement is equivalent to

$$
\left(\begin{array}{c}
\beta_{i j k 1} \\
\vdots \\
\beta_{i j k, r-1}
\end{array}\right)-T_{i}^{\prime} T_{j}^{\prime-1}\left(\begin{array}{c}
x_{j k 1} \\
\vdots \\
x_{j k, r-1}
\end{array}\right)+\left(\begin{array}{c}
x_{i k 1} \\
\vdots \\
x_{i k, r-1}
\end{array}\right)-(-1)^{t_{j}+t_{k}} h_{j k}\left(\begin{array}{c}
x_{i j 1} \\
\vdots \\
x_{i j, r-1}
\end{array}\right)=\left(\begin{array}{c}
0 \\
\vdots \\
0
\end{array}\right) .
$$

Looking separately to the $t_{i}$-th row and the others, the above equality is equivalent, by Lemma 5.1, to the vanishing of

$$
\begin{aligned}
\Lambda_{i j k}:= & \left(\begin{array}{c}
\beta_{i j k 1} \\
\vdots \\
\hat{\beta}_{i j k t_{i}} \\
\vdots \\
\beta_{i j k, r-1}
\end{array}\right)-P_{i j}\left(\begin{array}{c}
x_{j k 1} \\
\vdots \\
\hat{x}_{j k t_{j}} \\
\vdots \\
x_{j k, r-1}
\end{array}\right)- \\
& -(-1)^{t_{j}} x_{j k t_{j}} \Delta_{t_{i}} T_{i}^{\prime}\left(\begin{array}{c}
s_{j 1} \\
\vdots \\
s_{j, r-1}
\end{array}\right)+\left(\begin{array}{c}
x_{i k 1} \\
\vdots \\
\hat{x}_{i k t_{i}} \\
\vdots \\
x_{i k, r-1}
\end{array}\right)-(-1)^{t_{j}+t_{k}} h_{j k}\left(\begin{array}{c}
x_{i j 1} \\
\vdots \\
\hat{x}_{i j t_{i}} \\
\vdots \\
x_{i j, r-1}
\end{array}\right)
\end{aligned}
$$


and

$$
\begin{aligned}
& \lambda_{i j k}:=\beta_{i j k t_{i}}-\left(\delta_{i j 1} \ldots \hat{\delta}_{i j t_{j}} \ldots \delta_{i j, r-1}\right)\left(\begin{array}{c}
x_{j k 1} \\
\vdots \\
\hat{x}_{j k t_{j}} \\
\vdots \\
x_{j k, r-1}
\end{array}\right)- \\
& -(-1)^{t_{j}} s_{j t_{i}} x_{j k t_{j}}+x_{i k t_{i}}-(-1)^{t_{j}+t_{k}} h_{j k} x_{i j t_{i}}
\end{aligned}
$$

On the other hand, the condition $Z_{i k}^{\prime}-Z_{i j}^{\prime} Z_{j k}^{\prime}=0$ is equivalent, by Corollary 4.4, to the vanishing of $Q_{i k}^{\prime}-P_{i j} Q_{j k}^{\prime}-Q_{i j}^{\prime} S_{j k}^{\prime}$ and $S_{i k}^{\prime}-R_{i j} Q_{j k}^{\prime}-S_{i j}^{\prime} S_{j k}^{\prime}$. A straightforward calculation (using Lemmas 4.1 and 4.3 and Corollary 4.4) shows that

$$
Q_{i k}^{\prime}-P_{i j} Q_{j k}^{\prime}-Q_{i j}^{\prime} S_{j k}^{\prime}=\Lambda_{i j k}\left(g_{k},-f_{k}\right)
$$

and

$$
S_{i k}^{\prime}-R_{i j} Q_{j k}^{\prime}-S_{i j}^{\prime} S_{j k}^{\prime}=\lambda_{i j k}\left(\begin{array}{c}
f_{i} \\
g_{i}
\end{array}\right)\left(g_{k},-f_{k}\right)
$$

so that the lemma follows at once.

Remark 5.3. Equality (17) means that the $(r-1)$-uples

$$
(-1)^{t_{k}}{T_{i}^{\prime}}^{-1}\left(\begin{array}{c}
\beta_{i j k 1} \\
\vdots \\
\beta_{i j k, r-1}
\end{array}\right)
$$

represent a 2-coboundary in the Čech cohomology of $(r-1) L^{*}$ with respect to the covering $\left\{U_{i}\right\}_{i \in I}$ (multiplication by $h_{j k}$ in the last summand is needed in order to have all the $(r-1)$-uples defined in the trivialization of $(r-1) L^{*}$ in $\left.U_{k}\right)$. Recall (see $[9$, III-Theorem 4.5]) that the cohomology of coherent sheaves (and in particular of vector bundles) is isomorphic to the Čech cohomology of any affine cover. Hence the matrices $Z_{i j}^{\prime}$ will be the transition matrices of a vector bundle as soon as we see that the $(r-1)$-uples

$$
(-1)^{t_{k}}{T_{i}^{\prime}}^{-1}\left(\begin{array}{c}
\beta_{i j k 1} \\
\vdots \\
\beta_{i j k, r-1}
\end{array}\right)
$$

represent a 2-cocycle, since we are assuming $H^{2}\left(X,(r-1) L^{*}\right)=0$. This is what we are going to do next. 
Proposition 5.4. The set of $(r-1)$-uples

$$
(-1)^{t_{k}}{T_{i}^{\prime-1}}^{-1}\left(\begin{array}{c}
\beta_{i j k 1} \\
\vdots \\
\beta_{i j k, r-1}
\end{array}\right)
$$

defines a 2-cocycle of the vector bundle $(r-1) L^{*}$.

Proof. We need to show that for each $i, j, k, l \in I$, it follows

$$
\begin{gathered}
(-1)^{t_{l}}{T_{j}^{\prime}}^{-1}\left(\begin{array}{c}
\beta_{j k l 1} \\
\vdots \\
\beta_{j k l, r-1}
\end{array}\right)-(-1)^{t_{l}}{T_{i}^{\prime}}^{-1}\left(\begin{array}{c}
\beta_{i k l 1} \\
\vdots \\
\beta_{i k l, r-1}
\end{array}\right)+ \\
+(-1)^{t_{l}} T_{i}^{\prime-1}\left(\begin{array}{c}
\beta_{i j l 1} \\
\vdots \\
\beta_{i j l, r-1}
\end{array}\right)-(-1)^{t_{k}} h_{k l} T_{i}^{\prime-1}\left(\begin{array}{c}
\beta_{i j k 1} \\
\vdots \\
\beta_{i j k, r-1}
\end{array}\right)=\left(\begin{array}{c}
0 \\
\vdots \\
0
\end{array}\right) .
\end{gathered}
$$

As in the proof of Proposition 5.2, multiplying to the left by $(-1)^{t_{l}} T_{i}^{\prime}$ and applying Lemma 5.1, the above equality is equivalent to the vanishing of

$$
\begin{aligned}
\Delta_{i j k l}:=P_{i j}\left(\begin{array}{c}
\beta_{j k l 1} \\
\vdots \\
\hat{\beta}_{j k l t_{j}} \\
\vdots \\
\beta_{j k l, r-1}
\end{array}\right)+(-1)^{t_{j}} \beta_{j k l t_{j}} \Delta_{t_{i}} T_{i}^{\prime}\left(\begin{array}{c}
s_{j 1} \\
\vdots \\
s_{j, r-1}
\end{array}\right)- \\
-\left(\begin{array}{c}
\beta_{i k l 1} \\
\vdots \\
\hat{\beta}_{i k l t_{i}} \\
\vdots \\
\beta_{i k l, r-1}
\end{array}\right)+\left(\begin{array}{c}
\beta_{i j l 1} \\
\vdots \\
\hat{\beta}_{i j k t_{i}} \\
\vdots \\
\beta_{i j l, r-1}
\end{array}\right)-(-1)^{t_{k}+t_{l}} h_{k l}\left(\begin{array}{c}
\beta_{i j k 1} \\
\vdots \\
\hat{\beta}_{i j k t_{i}} \\
\vdots \\
\beta_{i j k, r-1}
\end{array}\right)
\end{aligned}
$$

and

$$
\begin{array}{r}
\lambda_{i j k l}:=\left(\delta_{i j 1} \ldots \hat{\delta}_{i j t_{j}} \ldots \delta_{i j, r-1}\right) \\
\left(\begin{array}{c}
\beta_{j k l 1} \\
\vdots \\
\hat{\beta}_{j k l t_{j}} \\
\vdots \\
\beta_{j k l, r-1}
\end{array}\right)+ \\
+(-1)^{t_{j}} \beta_{j k l t_{j}} s_{j t_{i}}-\beta_{i k l t_{i}}+\beta_{i j l t_{i}}-(-1)^{t_{k}+t_{l}} h_{k l} \beta_{i j k t_{i}} .
\end{array}
$$


To prove those equalities, we use the equality

$$
Z_{i j}\left(Z_{j l}-Z_{j k} Z_{k l}\right)-\left(Z_{i l}-Z_{i k} Z_{k l}\right)+\left(Z_{i l}-Z_{i j} Z_{j l}\right)-\left(Z_{i k}-Z_{i j} Z_{j k}\right) Z_{k l}=0 .
$$

Using Corollary 4.4 to split the above equality in two blocks -the one of the first $r-2$ rows and the one of the last 2 rows- and applying then Lemma 4.1, we get that the equality is equivalent to the vanishing of the matrices $\Lambda_{i j k l}\left(g_{l},-f_{l}\right)$ and $\lambda_{i j k l}\left(\begin{array}{c}f_{i} \\ g_{i}\end{array}\right)\left(g_{l},-f_{l}\right)$ which proves the proposition.

Remark 5.5. Although I did not check it, it is natural to expect that the map $\psi$ in (3) assigns to the morphism defined by $s_{1}, \ldots, s_{r-1}$ the cocycle of Proposition 5.4.

We finally prove the uniqueness statement.

Proposition 5.6. Assume $E$ is a vector bundle on $X$ satisfying conditions (i) and (ii) in Theorem 1.1. If $H^{1}\left(X, L^{*}\right)=0$, then any other vector bundle $E^{\prime}$ satisfying the same conditions is isomorphic to $E$.

Proof. Assume that the transition matrices of $E$ and $E^{\prime}$ are (see Lemma 4.1) respectively

$$
Z_{i j}=\left(\begin{array}{ll}
P_{i j} & Q_{i j} \\
R_{i j} & S_{i j}
\end{array}\right)
$$

and

$$
Z_{i j}^{\prime}=\left(\begin{array}{ll}
P_{i j} & Q_{i j}^{\prime} \\
R_{i j} & S_{i j}^{\prime}
\end{array}\right)
$$

with (see Remark 4.5)

$$
Q_{i j}^{\prime}=Q_{i j}+\left(\begin{array}{c}
x_{i j 1} \\
\vdots \\
\hat{x}_{i j t_{i}} \\
\vdots \\
x_{i j, r-1}
\end{array}\right)\left(g_{j},-f_{j}\right)
$$

and

$$
S_{i j}^{\prime}=S_{i j}+x_{i j t_{i}}\left(\begin{array}{c}
f_{i} \\
g_{i}
\end{array}\right)\left(g_{j},-f_{j}\right) .
$$

By Proposition 5.2, we have

$(-1)^{t_{k}} T_{j}^{\prime-1}\left(\begin{array}{c}x_{j k 1} \\ \vdots \\ x_{j k, r-1}\end{array}\right)-(-1)^{t_{k}} T_{i}^{\prime-1}\left(\begin{array}{c}x_{i k 1} \\ \vdots \\ x_{i k, r-1}\end{array}\right)+(-1)^{t_{j}} h_{j k} T_{i}^{\prime-1}\left(\begin{array}{c}x_{i j 1} \\ \vdots \\ x_{i j, r-1}\end{array}\right)=\left(\begin{array}{c}0 \\ \vdots \\ 0\end{array}\right)$, 
i.e., the $(r-1)$-uples

$$
(-1)^{t_{j}}{T_{i}^{\prime-1}}^{-1}\left(\begin{array}{c}
x_{i j 1} \\
\vdots \\
x_{i j, r-1}
\end{array}\right)
$$

define a 1-cocycle in $(r-1) L^{*}$. Since $H^{1}\left(X, L^{*}\right)=0$, this cocycle is the coboundary of a 0 -chain defined by $(r-1)$-uples that we write in the form

$$
(-1)^{t_{i}}{T_{i}^{\prime-1}}^{-1}\left(\begin{array}{c}
y_{i 1} \\
\vdots \\
x_{i, r-1}
\end{array}\right)
$$

This means

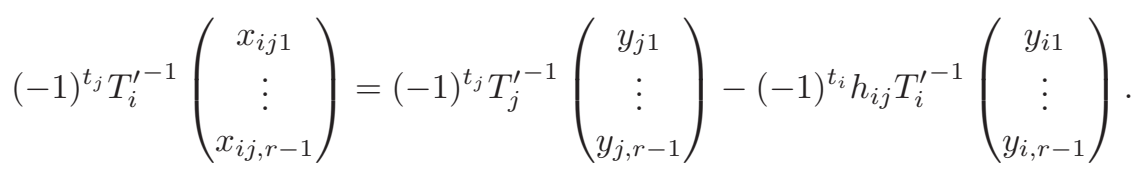

Multiplying as usual the above relation to the left by $(-1)^{t_{j}} T_{i}^{\prime}$ and applying Lemma 5.1 we get that this equality is equivalent to the vanishing of

$\Lambda_{i j}:=\left(\begin{array}{c}x_{i j 1} \\ \vdots \\ \hat{x}_{i j t_{i}} \\ \vdots \\ x_{i j, r-1}\end{array}\right)-P_{i j}\left(\begin{array}{c}y_{j 1} \\ \vdots \\ \hat{y}_{j t_{j}} \\ \vdots \\ y_{j, r-1}\end{array}\right)-(-1)^{t_{j}} y_{j t_{j}} \Delta_{t_{i}} T_{i}^{\prime}\left(\begin{array}{c}s_{j 1} \\ \vdots \\ s_{j, r-1}\end{array}\right)+(-1)^{t_{i}+t_{j}} h_{i j}\left(\begin{array}{c}y_{i 1} \\ \vdots \\ \hat{y}_{i t_{i}} \\ \vdots \\ y_{i, r-1}\end{array}\right)$

and

$$
\lambda_{i j}:=x_{i j t_{i}}-\left(\delta_{i j 1} \ldots \hat{\delta}_{i j t_{j}} \ldots \delta_{i j, r-1}\right)\left(\begin{array}{c}
y_{j 1} \\
\vdots \\
\hat{y}_{j t_{j}} \\
\vdots \\
y_{j, r-1}
\end{array}\right)-(-1)^{t_{j}} s_{j t_{i}} y_{j t_{j}}+(-1)^{t_{i}+t_{j}} h_{i j} y_{i t_{i}} .
$$

We consider the matrix

$$
N_{i}=\left(\begin{array}{cc}
I_{r-2} & N_{i}^{\prime} \\
0 & I_{2}+N_{i}^{\prime \prime}
\end{array}\right)
$$


where

$$
N_{i}^{\prime}=\left(\begin{array}{c}
y_{i 1} \\
\vdots \\
\hat{y}_{i t_{i}} \\
\vdots \\
y_{i, r-1}
\end{array}\right)\left(g_{i},-f_{i}\right)
$$

and

$$
N_{i}^{\prime \prime}=y_{i t_{i}}\left(\begin{array}{c}
f_{i} \\
g_{i}
\end{array}\right)\left(g_{i},-f_{i}\right)
$$

We define, for each $i \in I$, the automorphism of the trivial vector bundle $U_{i} \times k^{r}$ consisting of the multiplication by $N_{i}$ (observe that $\operatorname{det} N_{i}=1$ ). The result will be proved if we can patch all these automorphism in order to get an isomorphism between $E$ and $E^{\prime}$. For this, we need to check the equality $Z_{i j} N_{j}=N_{i} Z_{i j}^{\prime}$. Splitting this equality in four blocks, it becomes equivalent to two tautologies (using Lemma 4.3 (ii)) and the two equalities:

$$
P_{i j} N_{j}^{\prime}+Q_{i j}+Q_{i j} N_{j}^{\prime \prime}=Q_{i j}^{\prime}+N_{i}^{\prime} S_{i j}^{\prime}
$$

and

$$
R_{i j} N_{j}^{\prime}+S_{i j}+S_{i j} N_{j}^{\prime \prime}=S_{i j}^{\prime}+N_{i}^{\prime \prime} S_{i j}^{\prime}
$$

Using (18), (19), (20), (21), and Lemmas 4.1 and 4.3, these two equalities become respectively equivalent to the vanishing of $\Lambda_{i j}\left(g_{j},-f_{j}\right)$ and $\lambda_{i j}\left(\begin{array}{l}f_{i} \\ g_{i}\end{array}\right)\left(g_{j},-f_{j}\right)$, which completes the proof.

Remark 5.7. It is not by chance that $N_{i}$ takes the aspect obtained in the previous proof. It can be easily proved that this is the aspect that should take any matrix satisfying $N_{i} M_{i}=M_{i}$ and $\operatorname{det} N_{i}=1$. In other words, $N_{i}$ preserves the local expression of the sections $\alpha_{1}, \ldots, \alpha_{r-1}$ and the determinant of the transition matrix. This means that the isomorphism that we found preserves also the sections $\alpha_{1}, \ldots, \alpha_{r-1}$.

\section{Final remarks}

The natural question when trying to generalize Hartshorne-Serre construction to higher codimension is:

Question 6.1. Given a local complete intersection subscheme $Y$ of codimension $s$ of a smooth variety $X$, when is it possible to describe $Y$ as the dependency locus of $r-s+1$ sections of a rank $r$ vector bundle $E$ over $X$ ? When is it possible to take $r=s$ ? 
If one tries to imitate the technique explained in section 1, one can regard $Y$ as the degeneracy locus of a map $V \otimes \mathcal{O}_{X} \rightarrow E$, where $V$ is a vector space of dimension $r-s+1$. The Eagon-Northcott complex associated to this map produces a long exact sequence, analog to (1),

$$
0 \rightarrow S^{s-1} V \otimes \mathcal{O}_{X} \rightarrow S^{s-2} V \otimes E \rightarrow \cdots \rightarrow \bigwedge^{s-1} E \rightarrow \mathcal{J} \otimes L \rightarrow 0
$$

where $L=\bigwedge^{r} E$. Dualizing (22), using the isomorphism $\mathcal{E} x t^{s-1}\left(\mathcal{J}, \mathcal{O}_{X}\right) \cong \bigwedge^{s} N$ we get an epimorphism

$$
S^{s-1} V^{*} \otimes \mathcal{O}_{X} \rightarrow \bigwedge^{s} N \otimes L^{*}
$$

When trying to obtain (23) from (22), as in section 1 we get that the surjection provides an element of $\operatorname{Ext}^{s-1}\left(\mathcal{J} \otimes L, S^{s-1} V \otimes \mathcal{O}_{X}\right)$. An element there represents the class of a long exact sequence of length $s-1$ starting and finishing as (22), but if $s>2$ the equivalence classes of these extensions are difficult to deal with, and it does not look easy to decide when there is some equivalence class corresponding to an Eagon-Northcott complex like (22).

Unfortunately, our construction does not seem to give a hint to answer Question 6.1 when $s>2$ neither. Even when $r=s$ (i.e., when we want $Y$ to be the zero locus of a section of a vector bundle of rank $s$ ), our construction seems to suggest that everything could works as soon as $\bigwedge^{2} N$ is extendable to $X$, but this is a very strong condition. (For example, if $s=3$ this is essentially equivalent to say that $N$ itself is extendable, which is precisely what we want to prove.)

Observe also that, in the codimension two case, Hartshorne-Serre correspondence is saying (except for the cohomological condition on $L$ ) that a local complete intersection subscheme is the zero locus of a section of as rank two vector bundle if and only if the Chern classes of the normal bundle $N$ extend to the ambient variety. (The extendability of the second Chern class always holds by the self-intersection formula.) However, in higher codimension, although this condition is clearly necessary (since $N$ itself has to extend to the ambient variety) is not at all sufficient. (For instance, most of the elliptic curves in $\mathbb{P}^{4}$ will provide a counterexample.) Hence some extra condition is needed (see [4] for a discussion of codimension-three subvarieties in $\mathbb{P}^{3}$ and $\left.\mathbb{P}^{4}\right)$.

I finally want to mention that we expect that our construction could be generalized to other context different from algebraic varieties over an algebraically closed field. For instance, property (4) still holds in the context of real varieties (algebraic or not): it is enough to take $u=\frac{f}{f^{2}+g^{2}+1}$ and $v=\frac{g}{f^{2}+g^{2}+1}$ (I thank Marco Castrillón for suggesting me this idea); hence the whole construction seems to work in this new context. 


\section{References}

[1] A. Altman and S. Kleiman, Introduction to Grothendieck duality theory, Lecture Notes in Mathematics, vol. 146, Springer-Verlag, Berlin, 1970.

[2] E. Arrondo, La correspondencia de Serre hecha a mano, Homenaje al profesor Enrique Outerelo Domínguez, Contribuciones Matemáticas, Editorial Complutense, Madrid, 2004, pp. 61-72.

[3] W. Barth and A. Van de Ven, A decomposability criterion for algebraic 2-bundles on projective spaces, Invent. Math. 25 (1974), 91-106.

[4] M.-C. Chang, On the zero sets of vector bundles, Chinese J. Math. 21 (1993), no. 2, 109-114.

[5] D. Eisenbud, S. Popescu, and C. Walter, Enriques surfaces and other non-Pfaffian subcanonical subschemes of codimension 3, Comm. Algebra 28 (2000), no. 12, 5629-5653. Special issue in honor of Robin Hartshorne.

[6] Lagrangian subbundles and codimension 3 subcanonical subschemes, Duke Math. J. 107 (2001), no. 3, 427-467.

[7] H. Grauert and G. Mülich, Vektorbündel vom Rang 2 über dem n-dimensionalen komplexprojektiven Raum, Manuscripta Math. 16 (1975), no. 1, 75-100 (German, with English summary).

[8] R. Hartshorne, Varieties of small codimension in projective space, Bull. Amer. Math. Soc. 80 (1974), 1017-1032.

[9] , Algebraic geometry, Graduate Texts in Mathematics, vol. 52, Springer-Verlag, New York, 1977.

[10] G. Horrocks, A construction for locally free sheaves, Topology 7 (1968), 117-120.

[11] C. Okonek, M. Schneider, and H. Spindler, Vector bundles on complex projective spaces, Progress in Mathematics, vol. 3, Birkhäuser, Boston, Mass., 1980.

[12] J. J. Rotman, An introduction to homological algebra, Pure and Applied Mathematics, vol. 85, Academic Press Inc., New York, 1979.

[13] J. P. Serre, Sur les modules projectifs, Séminaire Dubreil-Pisot (1960/61), Secr. Math. Paris, exposé 2, 1961.

[14] J. A. Vogelaar, Constructing vector bundles from codimension-two subvarieties, PhD Thesis, Leiden, 1978.

[15] C. H. Walter, Pfaffian subschemes, J. Algebraic Geom. 5 (1996), no. 4, 671-704. 